\title{
Rationale Indikation zur Transfusion von Erythrozytenkonzentraten
}

\author{
K. Janetzko ${ }^{1}$, W. Ebell ${ }^{2}$, M. Welte ${ }^{3}$
}

${ }^{1}$ Institut für Transfusionsmedizin und Immunologie Mannheim, DRK-Blutspendedienst Baden-Württemberg Hessen

2 Klinik für Pädiatrie m. S. Onkologie/Hämatologie/SZT, Charité, Universitätsmedizin Berlin

${ }^{3}$ Klinik für Anästhesiologie und operative Intensivmedizin, Klinikum Darmstadt GmbH, Darmstadt

\section{Lernziele}

$\nabla$

- Grundlagen der Sauerstoffversorgung, der Anämie und deren physiologische Kompensationsmechanismen

- Qualitätsparameter von Erythrozytenkonzentraten

- Indikatoren einer anämischen Hypoxie - „Transfusionstrigger“

- Therapeutische Ziele einer Erythrozytentransfusion

- Transfusionsindikationen in Abhängigkeit von der Grunderkrankung/der Patientenklientel

- Transfusionsassoziierte Nebenwirkungen

- Meldewege bei Auftreten von transfusionsassoziierten Nebenwirkungen

- Korrekte Dokumentation einer Transfusion

\section{Zusammenfassung}

$\nabla$

Die allogene Erythrozytentransfusion ist seit Jahren essenzieller Bestandteil der modernen Medizin. Sie ist die am schnellsten wirksame, einzige kausale und anhaltend wirksame Therapie der lebensbedrohlichen anämischen Hypoxie. Risiken der Erythrozytentransfusion, vor allem Virusinfektionen, konnten aufgrund sorgfältiger Spenderauswahl und moderner Virusnachweisverfahren minimiert werden. Unabhängig davon muss der Einsatz von Erythrozytenkonzentraten (EK) dem Gebot einer rationalen Indikation folgen. Die Indikation zur Erythrozytentransfusion wird maßgeblich von der individuellen Kapazität des Patienten bestimmt, einen anämisch bedingten Sauerstoffmangel zu kompensieren. Bei Unterschreiten eines kritischen Sauerstoffangebots treten klinische Symptome der anämischen Hypoxie auf. Diese „physiologischen Transfusionstrigger" sind streng genommen die definitiven Indikatoren für eine Erythrozytentransfusion. Sie sind jedoch unspezifisch und unter klinischen Bedingungen nicht immer eindeutig zu bestimmen. Daher müssen neben klinischen Symptomen der anämischen Hypoxie bei der Indikationsstellung zur Erythrozytentransfusion die aktuelle Hämoglobinkonzentration, der Allgemeinzustand und Erkrankungen (z.B. koronare oder zerebrovaskuläre Gefäßerkrankungen), welche die individuelle Fähigkeit des Patienten limitieren, eine Anämie zu kompensieren, mitbeachtet werden. Aktuelle klinische Studien belegen, dass bei moderater Anämie (Hb [Hämoglobin] $8-10 \mathrm{~g} / \mathrm{dl}$ ) die meisten Patienten hinsichtlich ihrer Langzeitprognose nicht von Erythrozytentransfusionen profitieren. Eine restriktive Indikationsstellung zur Erythrozytentransfusion wird daher empfohlen. Aufgrund klinischer Beobachtungsstudien scheint es jedoch beim Erwachsenen bisher nicht gerechtfertigt, grundsätzlich nur Erythrozytenkonzentrate unterhalb einer bestimmten Lagerungsdauer zu transfundieren.

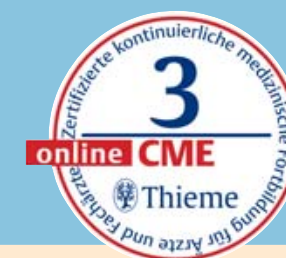
Schlüsselwörter
- Erythrozytenkonzentrate
- Anämie
- hypoxische Anämie
- Transfusionsindikation
- Transfusionsregime
- Lagerungsschäden bei Erythro- zytenkonzentraten
- Kompensationsmechanismen der Anämie

VNR

2760512013141213539

Bibliografie

DOI http://dx.doi.org/

10.1055/s-0032-1328134

Transfusionsmedizin 2013; 3: $31-48$

(c) Georg Thieme Verlag KG

Stuttgart · New York .

ISSN 2191-8805

Korrespondenzanschrift

Prof. Martin Welte

Klinik für Anästhesiologie und operative Intensivmedizin,

Klinikum Darmstadt GmbH

Grafenstraße 8

64383 Darmstadt

E-Mail: martin.welte@klinikum-

darmstadt.de 
- Erythrozytenkonzentrate werden aus frisch abgenommenem Vollblut oder ggf. maschinell mittels Zellseparatoren gewonnen.
- Die humane Ressource Blut wird knapper. Deshalb müssen Blutprodukte, insbesondere Erythrozytenkonzentrate, rational und nach Indikationskriterien eingesetzt werden.

- Die Transfusion von einem Erythrozytenkonzentrat führt bei einem Erwachsenen ohne Verbrauch zu einem Hämoglobinanstieg von ca. 1-1,5 g/dl bzw. Hämatokritanstieg von ca. $3-4 \%$.

\section{Erythrozytenkonzentrat (EK)}

Erythrozytenkonzentrate werden zumeist aus frisch abgenommenem Vollblut oder ggf. maschinell mittels Zellseparatoren gewonnen. Die durch das PEI (Paul-Ehrlich-Institut) zugelassenen Präparate unterscheiden sich geringfügig im Gehalt an noch verbleibenden Thrombozyten, Plasma und Additivlösung. Erythrozytenkonzentrate sind in Deutschland generell leukozytendepletiert ( Tab. 1), wodurch das Risiko einer Immunisierung gegen humane Leukozytenantigene (HLA) stark reduziert und die Übertragung zellständiger Viren (z.B. CMV [Zytomegalievirus]) weitgehend verhindert wurde. Die Transfusion von allogenem Vollblut ist in Deutschland obsolet.

Tab. 1 Qualitätsparameter für ein „Standarderythrozytenkonzentrat“ hergestellt aus Vollblut oder ggf. maschinell mittels Zellseparatoren [1].

\begin{tabular}{|l|l|}
\hline $\begin{array}{l}\text { Parameter } \\
\text { Volumen }\end{array}$ & gemäß der dem PEl angezeigten Spezifikation (ca. $240-330 \mathrm{ml})$ \\
\hline Hämatokrit & $50-70 \%$ \\
\hline Hämoglobin & $\geq 40 \mathrm{~g}$ Hämoglobin/Produkt \\
\hline Leukozyten & $<1 \times 10^{6} /$ Produkt \\
\hline Hämolyserate am Ende der Lagerungszeit & $<0,8 \%$ der Erythrozytenmasse \\
\hline Max. Lagerungszeit & $35-42$ Tage (je nach Zulassung) \\
& bei $4{ }^{\circ} \mathrm{C} \pm 2^{\circ} \mathrm{C}$ unter Einhaltung der Kühlkette \\
\hline
\end{tabular}

PEl: Paul-Ehrlich-Institut

\section{Verfügbarkeit von Erythrozytenkonzentraten}

In Deutschland wurden im Jahr 2010 insgesamt 6,123 $\times 10^{6}$ Blutprodukte transfundiert. Dabei belief sich die Anzahl der Plasmen auf $1,152 \times 10^{6}$, die der Thrombozytenkonzentrate auf $0,471 \times 10^{6}$ und die der Erythrozytenkonzentrate auf $4,5 \times 10^{6}$ [2]. Regelmäßige Blutspenden leisten aber nur ca. 3-4\% der Bevölkerung. Aufgrund der demografischen Entwicklung muss mit einem Rückgang der Blutspenderzahlen in den nächsten Jahren gerechnet werden. Im Vergleich dazu nimmt jedoch wegen der Alterung der Bevölkerung gerade die Altersgruppe, die am häufigsten medizinische Leistungen erhält, nämlich Menschen jenseits des 60. Lebensjahrs, zu.

\section{Auswahl und Dosierung von Erythrozytenkonzentraten}

Ein gesunder Erwachsener muss ca. $12 \mathrm{ml}$ Erythrozyten pro Tag produzieren, um die Hämoglobinkonzentration konstant bei $10 \mathrm{~g} / \mathrm{dl}$ (6,2 mmol/l) zu halten. Der Erythrozytenverbrauch ist bei vermehrtem Abbau, z. B. bei fieberhaften Erkrankungen, beim Vorliegen von Autoantikörpern und bei Splenomegalie, gesteigert ( $\bullet$ Tab. 2). Beim kompletten Ausfall der Erythrozytenproduktion, z. B. bei aplastischer Anämie, würde dies ca. 1 EK $(200-250 \mathrm{ml})$ pro Woche erfordern.

Die Transfusion von Erythrozytenkonzentraten erfolgt gemäß den Richtlinien [1] immer blutgruppengleich. Im Notfall wird eine Versorgung mit Erythrozytenkonzentraten der Blutgruppe 0 durchgeführt, bis die Patientenblutgruppe vorliegt [3] (๑ Tab. 3).

Tab. 2 In-vivo-Ergebnisse nach der Transfusion von Erythrozyten.

\begin{tabular}{ll}
$\begin{array}{l}\text { Klinische Ergebnisse } \\
\text { Recovery } 24 \text { h nach Transfusion }\end{array}$ & $>75 \%$ \\
\hline Mittlere Überlebensrate & ca. 60 Tage $(110-120$ Tage; Eliminationsrate $<1 \% /$ Tag $)$ \\
\hline Hb-Anstieg nach Transfusion* & ca. $1-1,5 \mathrm{~g} / \mathrm{dl}(0,62 \mathrm{mmol} / \mathrm{l})$ \\
\hline Hkt-Anstieg nach Transfusion* & ca. $3-4 \%$
\end{tabular}

* ohne aktive Blutung; Hb: Hämoglobin; Hkt: Hämatokrit 
Tab. 3 Blutgruppenkompatibilitäten [1].

\begin{tabular}{ll} 
Blutgruppe des Patienten & Kompatible Erythrozytenkonzentrate \\
A (Anti-B) & A und 0 \\
\hline B (Anti-A) & B und 0 \\
\hline$A B$ (keine Isoagglutinine) & $A B, A, B$ und 0 \\
\hline 0 (Anti-A und Anti-B) & 0 \\
\hline
\end{tabular}

Für die Transfusion stehen neben dem Erythrozytenkonzentrat hergestellt aus Vollblut oder ggf. maschinell mittels Apherese, noch weitere Spezialpräparationen zur Verfügung ( Tab.4).

Tab. 4 Auswahl unterschiedlicher Erythrozytenkonzentratpräparationen [1].

- Erythrozytenkonzentrat hergestellt aus Vollblut oder ggf. maschinell mittels Zellseparatoren (Tab. 1)

- Erythrozytenkonzentrat, Anti-CMV negativ

- Erythrozytenkonzentrat, bestrahlt mit $30 \mathrm{~Gy}$

- kryokonserviertes Erythrozytenkonzentrat

- individuelle Rezeptur:

Babyportionierung (ca. $4 \times 60-70 \mathrm{ml}$ )

Erythrozytenkonzentrat, eingeengt - individueller Hämotokrit

Erythrozytenkonzentrat gewaschen

CMV: Zytomegalievirus

Bestrahlte Erythrozytenkonzentrate

Die Übertragung vermehrungsfähiger, immunkompetenter Lymphozyten mit Blutprodukten kann bei immunkompromittierten Patienten zu einer Graft-versus-Host-Reaktion (GvHR) führen. Zellhaltige Blutprodukte, die an solche Patienten verabreicht werden, müssen deshalb mit 30 Gy bestrahlt werden ( 0 Tab. 5 ).

Tab. 5 Indikation für die Gabe bestrahlter Erythrozytenkonzentrate [1].

Indikation für die Gabe bestrahlter Erythrozytenkonzentrate

- Zellhaltige Blutkomponenten aus gerichteten Blutspenden von Blutsverwandten

- HLA-ausgewählte Blutkomponenten

- Intrauterine oder Z.n. intrauteriner Transfusion

- Postpartale Austauschtransfusion

- Nachweis oder Verdacht auf primäre, T-Zell- oder kombinierte Immundefekte

- Patienten vor und während autologer Stammzell- bzw. Knochenmarkentnahme und mindestens 3 Monate nach autologer Transplantation

- Spender vor und während allogener Stammzell- bzw. Knochenmarkspende und Patienten mindestens 6 Monate nach allogener Stammzell- bzw. Knochenmarktransplantation

- Patienten mit GvHR nach allogener Transplantation

- Patienten mit Morbus Hodgkin und Non-Hodgkin-Lymphomen (alle Stadien)

- Patienten unter Purinanalogatherapie

GvHR: Transplantat-gegen-Wirt-Reaktion; HLA: humanes Leukozytenantigen

\section{Gewaschene Erythrozytenkonzentrate}

Gewaschene Erythrozytenkonzentrate sind nur bei Patienten indiziert, bei denen Antikörper gegen IgA (Immunglobulin A) oder andere Plasmaproteine nachgewiesen werden oder bei denen wiederholt schwere, unklare, nicht hämolytische Transfusionsreaktionen beobachtet wurden. Erfahrungsgemäß liegt die Indikation zur Transfusion dieser Präparate extrem selten vor.
- Im Fall einer Notfalltransfusion sollte immer eine Blutprobe vor der Transfusion für die Blutgruppenbestimmung abgenommen werden, damit schnellstmöglich eine blutgruppengleiche Transfusion erfolgen kann. 
- Die Indikation zur Transfusion von kryokonservierten Erythrozytenkonzentraten ist bei Patienten mit komplexen Antikörpergemischen oder mit Antikörpern gegen hochfrequente Blutgruppenmerkmale gegeben.
- Fehltransfusion und transfusionsassoziierte Volumenüberlastung stellen die häufigsten Komplikationen dar.

\section{Kryokonservierte Erythrozytenkonzentrate}

Kryokonservierte Erythrozytenkonzentrate werden in wenigen nationalen Zentren eingelagert. Die Indikation zur Transfusion dieser Erythrozytenkonzentrate ist gegeben bei Patienten mit komplexen Antikörpergemischen oder mit Antikörpern gegen hochfrequente Blutgruppenmerkmale.

\section{Zytomegalievirus-negativ getestete Erythrozytenkonzentrate}

Die in Deutschland bei allen Erythrozyten- und Thrombozytenkonzentraten durchgeführte Leukozytendepletion bewirkt eine Abreicherung von zellständigen, latenten CMV und damit eine Verringerung des Risikos einer transfusionsassoziierten CMV-Infektion bei Risikopatienten um ca. 90\%. Ob die Verwendung CMV-seronegativ getesteter Blutspender das Risiko einer ta-CMVInfektion (ta: transfusionsassoziiert) weiter reduzieren kann, kann derzeit nicht beantwortet werden. Aufgrund der relativ hohen Serokonversionsrate besteht bei CMV-negativ getesteten Blutspendern das Risiko, dass die Blutspende im diagnostischen Fenster stattgefunden hat.

\section{Nebenwirkungen von Bluttransfusionen}

Wie bei allen Arzneimitteln sind auch im Zusammenhang mit der Transfusion von Blutprodukten Nebenwirkungen beschrieben, allerdings hat sich deren Spektrum in den letzten Jahren gewandelt. Mit der Einführung zunächst der Antikörper-, später der Antigentestung und der NAT (Nukleinsäureamplifikationstechnik) in das Spenderscreening wurde das transfusionsassoziierte Infektionsübertragungsrisiko durch HIV (humanes Immundefizienzvirus), HBV (Hepatitis-BVirus) und HCV (Hepatitis-C-Virus) deutlich minimiert. Die Erhebung einer umfänglichen Spenderanamnese, die Einführung der Leukozytendepletion, die Testung auf leukozytäre Antikörper nach Schwangerschaft und die aktuell immer umfangreicher durchgeführte Testung der Blutprodukte auf Bakterien tragen ebenfalls dazu bei, die Nebenwirkungsraten zu minimieren. Eine Übersicht geben die Meldungen der schwerwiegenden Transfusionszwischenfallreaktionen an die Bundesoberbehörde (PEI) ( $\bullet$ Abb. 1).

Somit stellen heute „vermeidbare Nebenwirkungen“ im direkten Zusammenhang mit der Anwendung von Blutprodukten, wie die Fehltransfusion und die transfusionsassoziierte Volumenüberlastung (TACO), die häufigsten Komplikationen dar. Die Schulung der Anwender, Patienten nach Blutgruppen und Vorbefunden zu befragen, die Patientenidentitätssicherung, die rechtzeitige Bestimmung von Blutgruppe und irregulären, transfusionsmedizinisch relevanten Antikör-

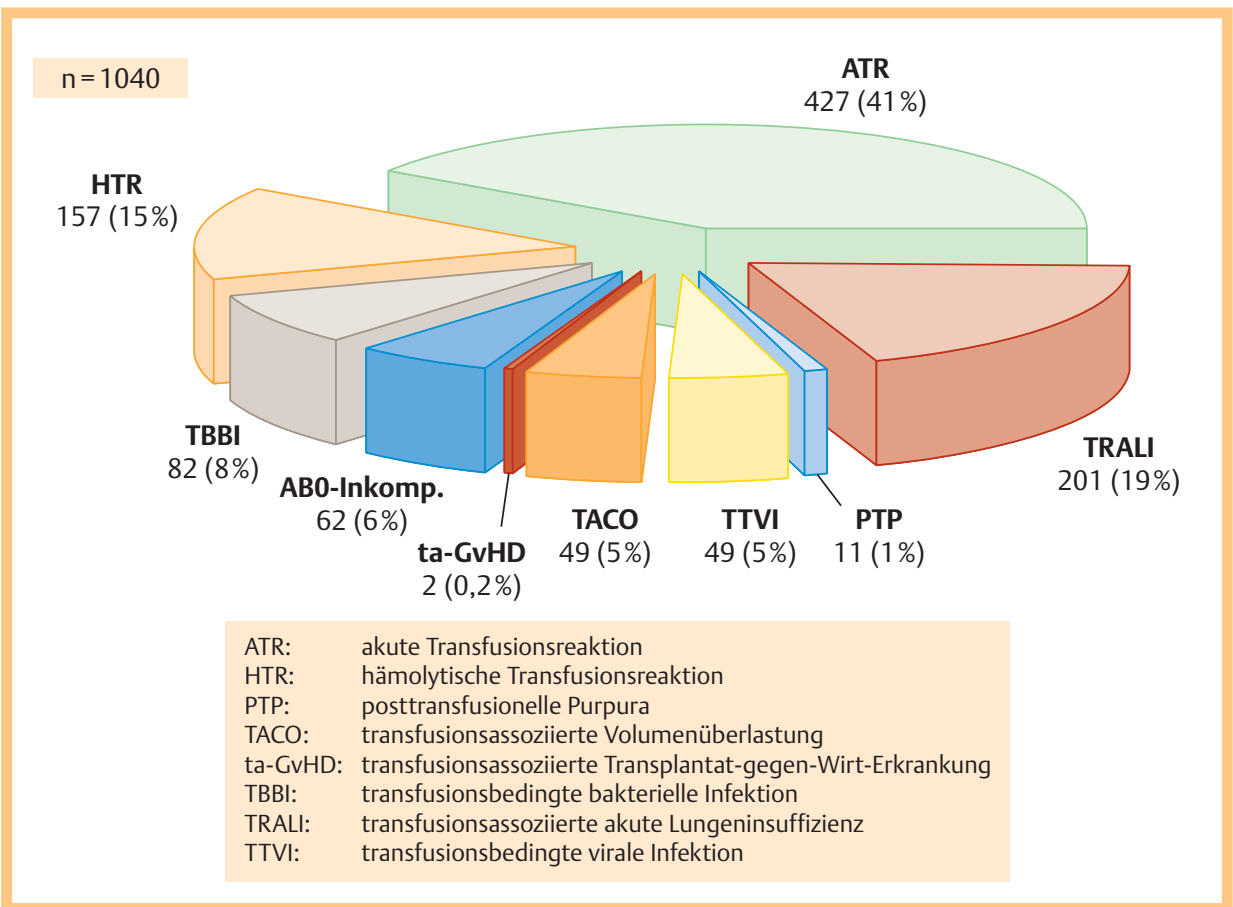

Abb. 1 Anzahl der dem Paul-Ehrlich-Institut gemeldeten schwerwiegenden Transfusionsreaktionen zwischen den Jahren 1997 - 2010 [2] bezogen auf 81,668 × $10^{6}$ transfundierte Blutprodukte: EK: 58,629 × 106 , TK: $5,190 \times 10^{6}$, FFP: $17,849 \times 10^{6}$.

EK: Erythrozytenkonzentrat; FFP: Fresh Frozen Plasma; TK: Thrombozytenkonzentrat 
pern im Vorfeld einer Transfusion sowie die korrekte Durchführung und Dokumentation des BST sind wichtige Maßnahmen, diese Anwendungsfehler zu minimieren.

Jedoch müssen ebenfalls Nebenwirkungen durch unbekannte Erreger oder solche, die erstmalig humanpathogen aufgetreten sind, berücksichtigt werden. Diese sog. „Re-Emerging Pathogens“ stellen immer ein potenzielles Risiko für eine transfusionsassoziierte Infektion dar. Ausgehend vom Zeitpunkt der Erkenntnis über eine „neue Infektion“, der Identifizierung des Pathogens bis hin zur Etablierung eines Testverfahrens kann längere Zeit vergehen. Beispiele hierzu sind der Wirtswechsel des HIV im Jahr 1981/1982, des HCV, identifiziert im Jahr 1989, des WestNil-Fieber-Virus im Jahr 1999 oder des Chikungunya-Virus im Jahr 2005. Die modernen molekularbiologischen Methoden (NAT) erlauben jedoch, nach Aufklärung des genetischen Codes, eine zunehmend schnellere Etablierung eines neuen Testverfahrens.

Ein weiteres Risiko geht von Erregern aus, für die es keine geeigneten Nachweisverfahren gibt z.B. Plasmodien, Leishmanien oder Trypanosomen. Allergische Reaktionen können ebenfalls nicht vermieden werden ( 0 Tab. 6).

Tab. 6 Unerwünschte Nebenwirkungen [3, 32].

\begin{tabular}{|c|c|}
\hline unerwünschte Nebenwirkungen & Risiko pro transfundierter Einheit \\
\hline \multicolumn{2}{|l|}{ transfusionsassoziierte Virusinfektionen } \\
\hline > HIV & $\begin{array}{l}\text { 1:4,3 Mio } \\
\text { diagnostische Fensterphase } 8-10 \text { Tage }^{*}\end{array}$ \\
\hline$>\mathrm{HBV}$ & $\begin{array}{l}\text { 1:360000 } \\
\text { diagnostische Fensterphase } 22 \text { Tage* }\end{array}$ \\
\hline$>\mathrm{HCV}$ & $\begin{array}{l}\text { 1: } 10,88 \text { Mio } \\
\text { diagnostische Fensterphase } 6-7 \text { Tage }^{*}\end{array}$ \\
\hline bakterielle Kontamination (Sepsis) & $1: 1 \times 10^{4}-1 \times 10^{5}$ \\
\hline übertragbare Form der Creutzfeldt-Jakob-Erkrankung (vCJK) & $\begin{array}{l}\text { Verdachtsfälle u. a. in Großbritannien; } \\
\text { keine Verdachtsfälle in Deutschland }\end{array}$ \\
\hline Parasitosen & Einzelfälle \\
\hline hämolytische Transfusionsreaktion, verzögert (Alloantikörper) & $1: 1 \times 10^{4}-1 \times 10^{5}$ \\
\hline tödlicher Ausgang & $1: 1 \times 10^{6}$ \\
\hline \multicolumn{2}{|l|}{ hämolytische Transfusionsreaktion } \\
\hline - Soforttyp (meist AB0, BG A auf 0) & $1: 1 \times 10^{4}-1 \times 10^{5}$ \\
\hline > tödlicher Ausgang & $1: 5-10 \times 10^{5}$ \\
\hline febrile, nicht hämolytische Transfusionsreaktion & $<1: 10^{3}$ \\
\hline $\begin{array}{l}\text { allergische Transfusionsreaktion } \\
\text { (IgE-AK des Empfängers, Anti-IgA-Mangel) }\end{array}$ & ca. $1: 200$ \\
\hline schwerer Verlauf & $1: 1 \times 10^{5}$ \\
\hline \multicolumn{2}{|l|}{ TRALI } \\
\hline - Plasma, Thrombozytenkonzentrate & $1: 1 \times 10^{4}-1 \times 10^{5}$ \\
\hline - Erythrozytenkonzentrate & $1: 1 \times 10^{6}$ \\
\hline TACO & bis $8 \%(1: 12,5)$ \\
\hline GvHD & Einzelfälle \\
\hline PTP & Einzelfälle \\
\hline
\end{tabular}

* Testung auf Antikörper und Virusgenom (PCR [Polymerasekettenreaktion])

BG: Blutgruppe; GvHD: Transplantat-gegen-Wirt-Erkrankung; HBV: Hepatitis-B-Virus; HCV: Hepatitis-C-Virus; HIV: humanes Immundefizienzvirus; IgA: Immunglobulin A; IgE-AK: Immunglobulin-E-Antikörper; PTP: posttransfusionelle Purpura; TACO: transfusionsassoziierte Volumenüberlastung; TRALI: transfusionsassoziierte akute Lungeninsuffizienz
- Obwohl Blutprodukte heute als so sicher wie nie gelten, muss ebenfalls vor dem Hintergrund der bleibenden transfusionsassoziierten Risiken die Indikation zur Transfusion immer kritisch abgewogen werden. 
- Bei gelagerten Erythrozyten kann es u. a. zu morphologischem Formwandel, Verminderung der Verformbarkeit und Abnahme des 2,3-DPG-Gehalts kommen.

- Die klinische Bedeutung der Lagerungsschäden kann derzeit nicht abschließend beurteilt werden. Unter Berücksichtigung der Tatsache, dass die klinischen Daten aus retrospektiven Kohortenstudien stammen und ein Teil dieser Studien vor Einführung der Leukozytendepletion (in Deutschland seit dem Jahr 2000) durchgeführt wurde, bleibt die Übertragbarkeit der Ergebnisse auf die heutige Situation unklar.

- Daher wird in den gültigen Leitlinien der Bundesärztekammer (BÄK) empfohlen, innerhalb der zugelassenen Lagerungsdauer nicht generell kurz gelagerte EK anzufordern.

\section{Lagerungseffekte}

Bei gelagerten Erythrozyten kommt es, abhängig von der Lagerungsdauer, zu Veränderungen (๑ Tab. 7) [4]:

- morphologischer Formwandel (z.B. Auftreten von Kugelzellen und Stechapfelformen) und Verminderung der Verformbarkeit

- Abnahme des 2,3-DPG-Gehalts (DPG: Diphosphoglycerat) mit Linksverschiebung der Sauerstoffdissoziationskurve

- Zunahme der Laktatkonzentration

- Freisetzung von erythrozytären Inhaltsstoffen (z. B. Kalium, Laktatdehydrogenase, Hämoglobin)

- Abnahme des S-Nitrosohämoglobins der Erythrozyten (Transportform des NO im Kreislauf) Diese sogenannten Lagerungsschäden sind z.T. nach der Transfusion in vivo innerhalb von 48-72 Stunden reversibel. Lagerungsschäden sind vermutlich für die Gewebeoxygenierung und den Verlauf der Erkrankung transfundierter Patienten von Bedeutung.

Klinische Studien zu den Auswirkungen der Lagerungsdauer auf die Gewebeoxygenierung kamen zu widersprüchlichen Ergebnissen. Assoziationen zwischen der Zunahme der Lagerungsdauer transfundierter Erythrozyten und der Mortalität, der Morbidität, der Inzidenz von Infektionen und multipler Organdysfunktionen sowie der Krankenhausliegedauer wurden bei retrospektiven Analysen nach Trauma und großen Operationen sowie bei kritisch kranken Erwachsenen festgestellt [5]. Im Gegensatz dazu fand die retrospektive Analyse aller Patienten, die in Dänemark und Schweden zwischen den Jahren 1995 und 2002 mindestens 1 Blutkonserve erhalten hatten, ein tendenziell erhöhtes Sterblichkeitsrisiko nur nach Transfusion von Erythrozytenkonzentraten, die länger als 30 Tage gelagert waren [6]. Eine Metaanalyse bestätigt dagegen einen Zusammenhang zwischen der Transfusion von länger gelagertem Blut und erhöhter Sterblichkeit [7]. Im Gegensatz dazu konnte jüngst in einer Studie an Patienten, die sich einer Hüft- oder Knieoperation unterziehen mussten, gezeigt werden, dass die Transfusion als solches mit einer erhöhten Mortalitätsrate einhergeht, dass jedoch innerhalb der Patientengruppe, die Erythrozytenkonzentrattransfusionen erhalten hatten, die Lagerungsdauer keine Einfluss auf die Mortalitätsrate hatte [8]. Als Ursache werden lagerungsbedingte Strukturveränderungen, Funktionsbeeinträchtigungen und die 2,3-DPG-Verarmung der Erythrozyten sowie die o.g. zellulären und bioaktiven Bestandteile im Plasmaüberstand, die eine immunmodulatorische Wirkung haben, diskutiert. Eine ganz aktuelle, retrospektive, an 6994 erwachsenen operativen Patienten mit unterschiedlichen Grunderkrankungen durchgeführte Auswertung zeigte ebenfalls

Tab. 7 Lagerungsschäden bei Erythrozytenkonzentraten [4].

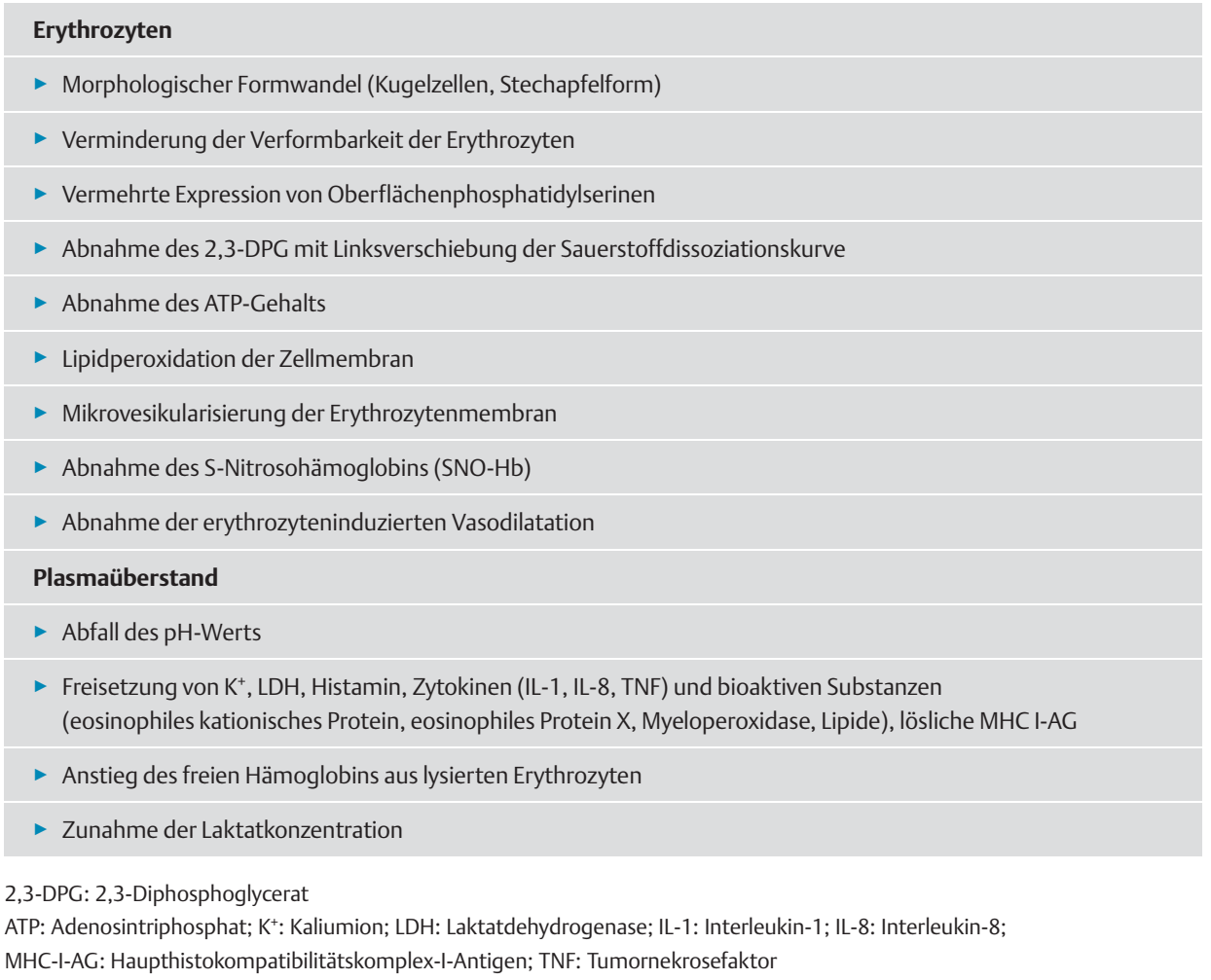


keine Assoziation zwischen Lagerungsalter der transfundierten Erythrozytenkonzentrate und postoperativer Mortalität (Hazard Ratio 0,99 [0,94-1,04]; p =0,64) [9].

Eine Tumorrezidive und Metastasierung begünstigende Wirkung gelagerter EK wird kontrovers diskutiert, ohne dass derzeit eine abschließende Bewertung möglich ist [10].

Die Tatsache, dass trotz ständiger Optimierungsschritte bei Auswahl, Herstellung und Lagerung von Blutkomponenten dennoch bei der Transfusion immer ein, wenn auch geringes, gesundheitliches Restrisiko für den Patienten bleibt, und die Tatsache, dass die Ressource Blut limitiert ist, erfordern einen verantwortungsvollen Umgang mit Blutprodukten. Eine Auswertung aus Österreich [11] lässt den Eindruck aufkommen, dass trotz bestehender Leitlinien die Transfusionsinzidenz bei definierten, identischen Eingriffen (Hüft- und Knie-TEP [TEP: totale Endoprothese], Kolon- und aortokoronare Bypasschirurgie) und vergleichbaren Blutungsverlusten oft erheblich zwischen den Kliniken differieren.

Die Entscheidung zur Transfusion muss mit konkreter Zielsetzung - und damit nicht aus Intuition heraus - sondern, wenn immer möglich, evidenzbasiert d.h. rational getroffen werden. AuBerdem verlangen die aktuellen Richtlinien zur Hämotherapie und das Transfusionsgesetz die Dokumentation der der Transfusion zugrunde liegenden Indikation [1].

\section{Grundlagen der Sauerstoffversorgung und physiologische Kompensationsmechanismen bei Anämie \\ $\nabla$}

Die physiologische Hauptaufgabe der Erythrozyten ist der Sauerstofftransport zu den Geweben und der Abtransport von Kohlenstoffdioxid von den Geweben. Darüber hinaus dienen sie dem Transport von Protonen, Ionen, organischem Phosphat und Stickstoffmonoxid sowie der Aufrechterhaltung der Hämostase. Erythrozyten sind kernlos und haben einen Durchmesser von 7,5-8,7 $\mu \mathrm{m}$, ein Volumen von 80-100 fl und eine Oberfläche von $136 \mu \mathrm{m}^{2}$. Durch ihre bikonkave Form und Lipiddoppelschichtmembran können sie anschwellen und ihr Volumen fast verdoppeln, aufgrund ihrer Verformbarkeit jedoch ebenfalls Kapillaren mit einem Durchmesser von $2,8 \mu \mathrm{m}$ passieren. Unter physiologischen Ruhebedingungen werden beim normalgewichtigen Menschen ca. $1000 \mathrm{ml}$ Sauerstoff/min transportiert bei einem Verbrauch von nur ca. $200-300 \mathrm{ml} / \mathrm{min}$. Aufgrund dieser breiten Sauerstoffangebotsreserve kann, auch bei akutem Mehrverbrauch (z. B. körperlicher Anstrengung) oder bei einem Abfall der Hämoglobinkonzentration auf ${ }^{2} / 3$ des normalen Ausgangswerts (z. B. von $15 \mathrm{~g} / \mathrm{dl}$ auf $10 \mathrm{~g} / \mathrm{dl}$ ), die Sauerstoffversorgung der Gewebe aufrechterhalten werden ( $\bigcirc$ Tab. 8).

Dabei wird der Sauerstoffmehrverbrauch bzw. der verminderte Sauerstoffgehalt im Blut bei akuter Anämie durch Anstieg des Blutflusses (Herzzeitvolumen), Steigerung der Sauerstoffextraktion aus dem arteriellen Blut sowie Umverteilung der Durchblutung zwischen den Organsystemen und in der Mikrozirkulation kompensiert.

Besteht allerdings simultan mit der akuten Anämie außerdem eine akute Verminderung des Blutvolumens z.B. bei akuter Blutung bzw. im hämorrhagischen Schock, sind die Kompensationsmechanismen nicht mehr wirksam und es kommt frühzeitiger zu einer Hypoxie. Aus therapeutischer Sicht ist es daher zwingend, zwischen hypo- und normovolämischer Anämie zu differenzieren.

Bei chronischer Anämie treten langfristige Adaptationsmechanismen, wie die Steigerung der Erythrozytenbildung und die Zunahme der intraerythrozytären Konzentration an 2,3-DPG auf, wodurch die Sauerstoffaffinität des Hämoglobins abnimmt bzw. die Sauerstoffdissoziationskurve nach rechts verschoben wird. Darüber hinaus zeigen sich kardiovaskuläre Veränderungen, die dauerhaft eine Steigerung des Herzzeitvolumens sowie des Blutflusses ermöglichen [12] (० Tab. 9).

Tab. 8 Primäre Mechanismen zur Kompensation des verminderten Sauerstoffgehalts des Blutes bei akuter Anämie.
- Zunahme des Herzschlagvolumens
- Zunahme der Herzfrequenz
- Zunahme des Herzzeitvolumens
- Umverteilung des Blutflusses zwischen den Organsystemen und in der Mikrozirkulation
- Zunahme der Sauerstoffextraktion aus dem arteriellen Blut

- Die physiologische Hauptaufgabe der Erythrozyten ist der Sauerstofftransport zu den Geweben und der Abtransport von Kohlenstoffdioxid von den Geweben.

- Bei akutem Blutverlust können gesunde Individuen unter strikter Aufrechterhaltung der Normovolämie die globale Sauerstoffversorgung bis zu einer Hämoglobinkonzentration von circa $6 \mathrm{~g} / \mathrm{dl}$ (3,7 mmol/l) aufrechterhalten, ohne dass dauerhafte hypoxische Schäden entstehen.

- Bei chronischer Anämie treten langfristige Adaptationsmechanismen, z. B. Steigerung der Erythrozytenbildung und Zunahme der intraerythrozytären Konzentration an 2,3-DPG, auf. 
Tab.9 Primäre Mechanismen zur Kompensation des verminderten Sauerstoffgehalts des Blutes bei chronischer Anämie [12].

- Steigerung der Erythrozytenbildung

- Zunahme der intraerythrozytären Konzentration an 2,3-Diphosphoglycerat (2,3-DPG), wodurch die Sauerstoffaffinität des Hämoglobins abnimmt bzw. die Sauerstoffdissoziationskurve nach rechts verschoben wird

- kardiovaskuläre Veränderungen, die dauerhaft eine Steigerung des Herzzeitvolumens sowie des Blutflusses ermöglichen

Das Ausmaß der physiologischen Fähigkeit des Organismus, bei einer akuten Anämie den Verlust an Sauerstofftransportkapazität zu kompensieren, ist jedoch individuell unterschiedlich. Es wird von Alter, allgemeinem Gesundheitszustand, aktuellem klinischen Zustand, intravasalem Blutvolumen und Erkrankungen, die mit den primären physiologischen Kompensationsmechanismen interferieren (z.B. koronare oder zerebrovaskuläre Gefäßerkrankungen, Herzinsuffizienz) beeinflusst.

Erst nach Erschöpfung der physiologischen Kompensationsmechanismen kommt es zur Minderversorgung der Gewebe mit Sauerstoff, d. h. zur Hypoxie, die, wenn sie auf der kritischen Reduktion der Hämoglobinkonzentration beruht, als „anämische Hypoxie“ bezeichnet wird.

\section{Indikationen zur Transfusion bei akuter Anämie $\nabla$}

Bei der Indikationsstellung zur Erythrozytentransfusion müssen neben Faktoren, welche die physiologische Kompensationsfähigkeit bei Anämie limitieren, auch anämiespezifische Faktoren berücksichtigt werden. Hierzu zählen Ursache, zeitlicher Verlauf, Dauer und Schweregrad der Anämie.

Die individuell unterschiedliche, physiologische Kompensationsfähigkeit und die variable Dynamik der Anämie machen verständlich, dass die gemessene Hämoglobinkonzentration über weite Bereiche nicht mit der Ausprägung der klinischen Symptome der Anämie korreliert. Folglich kann die Hämoglobinkonzentration allein kein suffizientes Kriterium zur Diagnose einer anämischen Hypoxie bzw. der alleinige Transfusionstrigger sein. Das wird in den aktuellen Leitlinien ausdrücklich hervorgehoben [13].

Vielmehr müssen zur Indikationsstellung einer Transfusion bei laborchemisch gesicherter Anämie und Normovolämie zusätzliche klinische Symptome, die sog. „physiologischen Transfusionstrigger“, berücksichtigt werden, die auf eine anämische Hypoxie hinweisen können ( Tab. 10). Sie sind Indikatoren dafür, dass die Fähigkeit eines individuellen Organismus zur Kompensation einer Anämie erschöpft ist, und stellen somit - zumindest aus physiologischer Sicht - die definitive Indikation zur Erythrozytentransfusion dar.

An gesunden Probanden erhobene Befunde zeigen, dass unter normovolämischen Bedingungen die Sauerstoffversorgung erst bei einem Abfall der Hämoglobinkonzentration unter ca. $5 \mathrm{~g} / \mathrm{dl}$ kritisch vermindert wird [12].

Für kardiovaskuläre Risikopatienten mit koronarer Herzkrankheit, Herzinsuffizienz oder zerebrovaskulärer Erkrankung liegen keine ausreichenden Daten für eindeutige Empfehlungen vor. Auf Basis der vorhandenen Daten kann jedoch geschlossen werden, dass diese Patienten bei hämodynamischer Stabilität und ohne Anzeichen einer anämischen Hypoxie bei Hb-Konzentrationen zwischen 8 und $10 \mathrm{~g} / \mathrm{dl}$ nicht von Erythrozytentransfusionen profitieren, wie eine jüngst publizierte, große randomisierte und kontrollierte Untersuchung an älteren kardialen Risikopatienten (KHK [koronare Herzkrankheit] oder Risikofaktoren für KHK), die sich einer Hüftgelenksoperation unterzogen, zeigte [14].

Selbst Hämoglobinkonzentrationen von 7-8 g/dl (4,3-5,0 mmol/l, Hkt [Hämatokrit] 21 - 24\%) werden von kardiovaskulär stabilen Risikopatienten ohne bleibende hypoxische Schädigungen toleriert. Erst ein Absinken der Hämoglobinkonzentration unter $7 \mathrm{~g} / \mathrm{dl}(<4,3 \mathrm{mmol} / \mathrm{l})$ geht mit einer Zunahme der Morbidität und Mortalität einher [15].

Für die Versorgung von Schwer- und Mehrfachverletzten (Polytrauma) mit massiver Blutung empfiehlt die aktuelle S3-Leitlinie Polytrauma/Schwerverletztenbehandlung, dass die Therapie mit Blutprodukten entsprechend einem spezifischen Massivtransfusionsprotokoll erfolgen sollte [16]. Da Erythrozyten an der primären Hämostase beteiligt sind und sich eine signifikante Beeinträchtigung der Gerinnung deutlich vor einer Beeinflussung der Oxygenierung entwickelt, wird weiterhin empfohlen, dass bei aktiv blutenden Patienten die Indikation zur Erythrozytentransfusion bei Hb-Werten unter $10 \mathrm{~g} / \mathrm{dl}$ gestellt und der Hämatokrit bei 30\% gehalten werden sollte. 
Tab. 10 Physiologische Transfusionstrigger [3].

\author{
Kardiopulmonale Symptome \\ Tachykardie (z. B. > 110/min oder > 25\% über Ausgangswert) \\ Hypotension (art. Mitteldruck $<60 \mathrm{mmHg}$ o. Abfall $>25 \%$ ) \\ Blutdruckabfall unklarer Genese \\ Dyspnoe \\ Ischämietypische EKG-Veränderungen \\ Neu auftretende ST-Senkungen $(>0,2 \mathrm{mV})$ \\ Neu auftretende ST-Hebungen $>0,2 \mathrm{mV}$ \\ Neu auftretende Rhythmusstörungen \\ Neue myokardiale Kontraktionsstörungen im Echokardiogramm \\ Globale Indices einer unzureichenden Sauerstoffversorgung \\ Anstieg der globalen $\mathrm{O}_{2}$-Extraktion $>50 \%$ \\ Abfall der $\mathrm{O}_{2}$-Aufnahme $>10 \%$ vom Ausgangswert \\ Abfall der gemischtvenösen $\mathrm{O}_{2}$-Sättiging $<50 \%$ \\ Abfall des gemischtvenösen $\mathrm{PO}_{2}<32 \mathrm{mmHg}$ \\ Abfall der zentralvenösen $\mathrm{O}_{2}$-Sättigung $<60 \%$ \\ Laktatazidose (Laktat $>2 \mathrm{mmol} / \mathrm{l}+$ Azidose) \\ $\mathrm{O}_{2}$ : Sauerstoff; $\mathrm{PO}_{2}$ : Sauerstoffdruck
}

Die für polytraumatisierte Patienten geltende Vorgehensweise sollte analog bei anderen Patienten mit aktiver, massiver und unkontrollierter Blutung angewendet werden. Neben der Transfusion von Erythrozyten muss bei diesen Patienten außerdem an die rechtzeitige Substitution von Gerinnungsfaktoren und Thrombozyten gedacht werden.

Auf Basis der verfügbaren Studiendaten wurden in den Leitlinien der Bundesärztekammer Empfehlungen zur Transfusion von Erythrozyten bei akuter Anämie unter Berücksichtigung der aktuellen Hämoglobinkonzentration, physiologischen Kompensationsmechanismen, vorhandener kardiovaskulärer Risikofaktoren und klinischen Hinweisen auf eine manifeste anämische Hypoxie (physiologische Transfusionstrigger) zusammengefasst ( 0 Tab. 10).

Unbestritten ist, dass in bestimmten Situationen wie z.B. bei aktiver Blutung und Zeichen der Hypoxie (physiologische Transfusionstrigger), im hämorrhagischen Schock, bei Unterschreiten eines, individuell allerdings schwer diagnostizierbaren, kritischen Hämoglobinwerts sowie bei Austauschtransfusionen des Neugeborenen die rechtzeitige Gabe von Erythrozytenkonzentraten absolut indiziert ist, um hypoxische Schäden zu vermeiden - zumindest wenn keine andere, mindestens gleichwertige Therapie zur Verfügung steht.

\section{Therapeutisches Ziel der Erythrozytentransfusion}

Bei der Indikationsstellung zur Erythrozytentransfusion wird implizit davon ausgegangen, dass durch die Transfusion der Krankheitsverlauf günstig beeinflusst wird. Je nach therapeutischem Ziel wird erwartet, dass durch die Transfusion die Gewebeoxygenierung verbessert, die Mortalität oder Morbidität vermindert, die Inzidenz kardiovaskulärer, zerebrovaskulärer oder pulmonaler Komplikationen günstig beeinflusst sowie das Infektionsrisiko gesenkt werden. Weitere Ziele sind, die Rekonvaleszenz zu beschleunigen oder die Lebensqualität zu verbessern. Nach heutigem Kenntnisstand ist jedoch vielfach unklar, ob überhaupt, in welcher Situation oder ab welchem Schweregrad einer Anämie diese therapeutischen Ziele durch die Erythrozytentransfusion erreicht werden können [17].

So ist eine Verbesserung der Gewebeoxygenierung durch die Transfusion von Erythrozyten nur dann zu erwarten, wenn eine bestehende Gewebehypoxie - zumindest teilweise - durch eine kritische Verminderung der Sauerstoffträger bedingt ist, d.h. eine „anämische“ Komponente hat. Dies trifft z. B. im hämorrhagischen Schock oder bei Unterschreiten des kritischen Hämatokrits zu. Liegt keine anämische Hypoxie(-komponente) vor, werden Erythrozyten die Gewebeoxygenierung nicht verbessern. Dies bestätigen Untersuchungen an Intensivpatienten, die bei moderater Anämie (Hb ca. 8,0-0,0 g/dl) keine Verbesserung der Sauerstoffaufnahme und Gewebeoxygenierung durch Erythrozytentransfusionen feststellten [18].
- Unbestritten ist, dass in bestimmten Situationen die rechtzeitige Gabe von Erythrozytenkonzentraten absolut indiziert ist, um hypoxische Schäden zu vermeiden. 
- Voraussetzung für die therapeutische Effizienz einer Erythrozytentransfusion ist das Vorliegen einer anämischen Hypoxie(-komponente). In diesem Fall führt die Gabe von Erythrozyten zu einer Verbesserung der Gewebeoxygenierung.
Ein positiver therapeutischer Effekt von Erythrozytentransfusionen auf die Sterblichkeit oder die Morbidität konnte in zahlreichen, allerdings meist retrospektiven Beobachtungsstudien an unterschiedlichen Patientengruppen (Trauma- und Intensivpatienten, herz-, gefäß- und allgemeinchirurgische Patienten) bisher nicht nachgewiesen werden [19]. Im Gegenteil: In der Mehrzahl der Studien hatten transfundierte im Vergleich zu nicht transfundierten Patienten eine schlechtere Prognose und höhere Komplikationsraten (Infektionen, Wundheilungsstörungen, pulmonale Komplikationen, verlängerte Liegedauer). Dabei scheint nicht nur die Massivtransfusion, sondern bereits die Gabe von wenigen Erythrozytenkonzentraten eine Relevanz zu besitzen. Bei herz- und allgemeinchirurgischen Patienten waren wenige perioperative Transfusionen ( $\leq 2$ Einheiten) mit einer erhöhten Sterblichkeit und einer höheren Inzidenz an Komplikationen assoziiert [19].

Einschränkend muss berücksichtigt werden, dass aufgrund des retrospektiven Studiendesigns („Selection-Bias“, d. h. nicht berücksichtigte Störfaktoren und dadurch bedingte nicht erkannte Imbalancen im untersuchten Patientengut sowie keine definierten Transfusionsindikationen) und der unterschiedlichen Qualität der verabreichten Blutprodukte (z. B. leukozytendepletiert vs. nicht depletiert, verschiedene Lagerungszeiten) trotz des scheinbar eindeutigen statistischen Zusammenhangs zwischen Erythrozytentransfusion und ungünstiger Prognose nicht sicher auf einen kausalen Zusammenhang geschlossen werden kann. Die Möglichkeit des „Selection-Bias“ durch nicht balancierte Störfaktoren unterstreicht eine aktuelle Studie [20]: Bei definierten chirurgischen Eingriffen wurden sowohl transfundierte mit nicht transfundierten Patienten als auch Zentren mit hoher und niedriger Transfusionsrate verglichen. Es bestätigte sich der negative Effekt der Transfusion auf die Sterblichkeit im direkten Patientenvergleich, nicht jedoch im Zentrenvergleich. Obwohl die Transfusionsraten sich zwischen den in unterschiedlichen Zentren operierten Patienten drastisch unterschieden, konnte kein Einfluss der Transfusion auf die Prognose festgestellt werden.

Festzuhalten bleibt daher, dass nach heutigem Kenntnisstand durch die Transfusion von Erythrozyten das angestrebte therapeutische Ziel häufig nicht erreicht wird; ob Transfusionen tatsächlich mit negativer Prognose assoziiert sind, bleibt offen.

\section{"Liberale“ vs. „restriktive“ Indikation zur Erythrozytentransfusion}

Aufgrund der Hinweise auf eine ungünstige Beeinflussung der Prognose durch Erythrozytentransfusionen wurden klinische Studien initiiert, in denen meist „Standard-Transfusionsindikationen“ ( $\mathrm{Hb}<10 \mathrm{~g} / \mathrm{dl}$ ) mit restriktiveren und die individuelle Physiologie berücksichtigenden Konzepten verglichen wurden. Prospektive, randomisierte und kontrollierte Studien an erwachsenen Intensivpatienten [21], an herzchirurgischen Patienten [22] und an älteren kardiovaskulären Risikopatienten [14] kommen übereinstimmend zu dem Ergebnis, dass bei moderater Anämie (Hb 7,0-10,0 g/dl) und ohne aktive Blutung die Transfusion von Erythrozytenkonzentraten auf Hb-Werte über 10,0 g/dl („liberale Transfusionsstrategie“) die Sterblichkeit und die Morbidität gegenüber „restriktiveren“ Transfusionsindikationen (Hb ca. 7,0-9,0 g/dl) nicht verbessert. Jüngere, unter 55 Jahre, oder weniger schwer erkrankte Intensivpatienten (APACHEScore [APACHE: Acute Physiology And Chronic Health] < 20 Punkte) überlebten signifikant häufiger, wenn die Indikation zur Transfusion zurückhaltend gestellt und moderat anämische HbWerte toleriert wurden [21].

Von besonderer klinischer Relevanz ist die Studie von Carson und Mitarbeitern (FOCUS-Trial). Sie verglich prospektiv an älteren (> 50 Jahre) kardialen Risikopatienten (KHK oder Risikofaktoren für KHK), die sich einer Hüftoperation unterzogen, ein liberales Transfusionsschema (Erythrozytentransfusionen bei einem $\mathrm{Hb}-\mathrm{Wert}<10 \mathrm{~g} / \mathrm{dl}$ ) mit einem restriktiven Tranfusionsschema (Erythrozytentransfusionen bei Symptomen einer Anämie bzw. bei einem Hb-Wert $<8 \mathrm{~g} / \mathrm{dl}$ ). Als klinische Symptome der Anämie galten: akute thorakale Schmerzen (V.a. Angina Pectoris), akute Herzinsuffizienz, Tachykardie und Hypotension. Die primären Studienendpunkte waren die Mortalität sowie die Fähigkeit, gehen zu können, beobachtet in einem postoperativen 60-Tage-Zeitraum [14] ( Tab. 11). Es zeigte sich, dass zwischen beiden randomisierten Patientengruppen kein Unterschied hinsichtlich Mortalität, der Inzidenz kardio- und zerebrovaskulärer Symptome oder der postoperativen körperlichen Belastbarkeit (der Fähigkeit, ohne fremde Hilfe laufen zu können) bestand. Während bei liberaler Indikationsstellung 97\% der Patienten mindestens 1 Transfusion erhielten, waren es in der restriktiven Gruppe nur 40\%.

Eine aktuelle Metaanalyse der Cochrane Library bestätigt die Ergebnisse der genannten klinischen Studien [23]. Eine restriktive Indikationsstellung zur Transfusion vermindert das Risiko der Blutübertragung (RR [relatives Risiko] 0,66; 95\%-KI [Konfidenzintervall] 24-45\%), die Anzahl der transfundierten Erythrozyteneinheiten (-1,19, 95\%-KI 0,53-1,85) und die Krankenhausmortalität (RR 0,77; 95\%-KI 0,62-0,95). Hinsichtlich der 30-Tage-Mortalität (RR 0,85; 95\%-KI 0,70-1,03), kardialer Komplikationen, Myokardinfarkte, thromboembolischer Ereignis- 
Tab. 11 Liberales vs. restriktives Transfusionsregime: Ergebnis 60 Tage nach der OP [14].

\begin{tabular}{|c|c|c|}
\hline Transfusionsindikation & liberales Transfusionsregime & $\begin{array}{l}\text { restriktives, symptomorien- } \\
\text { tiertes Transfusionsregime }\end{array}$ \\
\hline Anzahl der Patienten & 1007 & 1009 \\
\hline Hb vor Transfusion (g/dl) & $9,2 \pm 0,5$ & $7,9 \pm 0,6(p<0,001)$ \\
\hline \multicolumn{3}{|l|}{ Outcome 60 Tage post-OP } \\
\hline EK transfundiert & 1866 & 652 \\
\hline Sterblichkeit & $7,6 \%$ & $6,6 \%(p=0,34)$ \\
\hline unfähig zu laufen & $27,6 \%$ & $28,1 \%$ \\
\hline kardiale Komplikationen & $4,3 \%$ & $5,2 \%$ \\
\hline zerebrovaskuläre Komplikationen & $0,8 \%$ & $0,3 \%$ \\
\hline
\end{tabular}

Hb: Hämoglobin; EK: Erythrozytenkonzentrat

Tab. 12 Empfehlungen zur Transfusion von Erythrozytenkonzentraten bei Erwachsenen [3].

\begin{tabular}{|lll|}
\hline Hb-Bereich & Kompensationsfähigkeit/Riskofaktoren & $\begin{array}{l}\text { Eine Transfusion } \\
\text { sollte erfolgen: }\end{array}$ \\
\hline $\begin{array}{l}\leq 5 \mathrm{~g} / \mathrm{dl} \\
(\leq 3,1 \mathrm{mmol} / \mathrm{l})\end{array}$ & $\begin{array}{l}\text { (Hkt von ca. 15\%) muss bei normaler Herz-Kreislauf-Funktion } \\
\text { und Normovolämie als kritischer Grenzwert der absoluten Trans- } \\
\text { fusionsindikation angenommen werden! }\end{array}$ & ja \\
\hline$\leq 6 \mathrm{~g} / \mathrm{dl}$ & - & ja \\
$(\leq 3,7 \mathrm{mmol} / \mathrm{l})$ & & nein \\
\hline$>6-8 \mathrm{~g} / \mathrm{dl}$ & Kompensation adäquat, keine Risikofaktoren \\
$(>3,7-5,0 \mathrm{mmol} / \mathrm{l})$ & & ja \\
\hline$>6-8 \mathrm{~g} / \mathrm{dl}$ & Kompensation eingeschränkt, Risikofaktoren & \\
\hline$>6-8 \mathrm{~g} / \mathrm{dl}$ & (KHK, Herzinsuffizienz, zerebrovaskuläre Insuffizienz) & ja \\
\hline$<7-8 \mathrm{~g} / \mathrm{dl}$ & Hinweis auf anämische Hypoxie (Tachykardie, Hypotension, \\
$(<4,3-5,0 \mathrm{mmol} / \mathrm{l})$ & EKG-Ischämie, Laktatazidose) & ja \\
\hline$>10 \mathrm{~g} / \mathrm{dl}$ & Khronische Anämie & nein \\
$(\geq 6,2 \mathrm{mmol} / \mathrm{l})$ & & \\
\hline
\end{tabular}

EKG: Elektrokardiogramm; Hkt: Hämatokrit; KHK: koronare Herzkrankheit

se, der funktionellen Erholung (Rekonvaleszenz), der Inzidenz von Pneumonien und der Liegedauer (Intensivstation, Krankenhaus) sind restriktive Transfusionsstrategien den liberaleren ebenbürtig.

Aufgrund dieser Datenlage empfehlen die aktuellen Leitlinien, bei Patienten ohne spezifische kardio- oder zerebrovaskuläre Risiken und ohne aktive Blutung die Indikation zur Transfusion von Erythrozyten restriktiv zu stellen und keine Normalisierung der Hämoglobinkonzentration anzustreben (० Tab. 12).

\section{Indikation zur Transfusion bei chronischer Anämie}

$\nabla$

Grundsätzlich wird eine langsam entstehende, chronische Anämie besser toleriert als eine akute. Patienten ohne kardiovaskuläre Erkrankungen können bei Normovolämie extrem niedrige Hämoglobin- und Hämatokritwerte gut kompensieren. Dennoch kann eine chronische Anämie den klinischen Verlauf einer Erkrankung verschlechtern (z. B. bei Herzinsuffizienz) und die Mortalitätsraten erhöhen [24]. Durch Anheben der Hämoglobinkonzentration kann die objektive Belastbarkeit und das subjektive Wohlbefinden betroffener Patienten mit chronischer Anämie verbessert sowie die Rate an stationären Behandlungen reduziert werden.

Bei Patienten mit chronischer, renaler Anämie verbesserte die Normalisierung der Hämoglobinkonzentration auf Werte von $13-15 \mathrm{~g} / \mathrm{dl}$ gegenüber moderat anämischen Werten von $11-13 \mathrm{~g} / \mathrm{dl}$

- Abhängig von der Patientenklientel geht ein restriktives Transfusionsschema mit keinen gesundheitlichen Nachteilen und möglicherweise für bestimmte Patienten sogar mit Vorteilen einher. 
- Bei Patienten mit chronischer Anämie (Hämoglobinkonzentration $<8-7 \mathrm{~g} / \mathrm{dl}[<5,0-4,3 \mathrm{mmol} / \mathrm{ll}]$ sollten EK transfundiert werden.

- Gemäß der aktuellen Literatur ist bei Kindern die Erythrozytentransfusion mit einem höheren Morbiditäts- und Mortalitätsrisiko assoziiert, sodass als Transfusionstrigger ein $\mathrm{Hb}$-Wert von $7 \mathrm{~g} / \mathrm{dl}$ empfohlen wird. Hiervon ausgenommen sind Früh- und Neugeborene. Die Präparate sollten nicht länger als 3 Wochen gelagert sein. zwar die subjektive Lebensqualität, die Inzidenz von anämiebedingten Komplikationen (z.B. kardiovaskulär) wurde dadurch aber nicht weiter reduziert [25].

Kardiovaskuläre Risikopatienten (koronare oder zerebrovaskuläre Gefäßerkrankung, Herzinsuffizienz) mit chronischer Anämie profitieren von höheren Hämoglobinkonzentrationen. So werden insbesondere bei schwerer Herzinsuffizienz das Überleben, die Belastungsfähigkeit und die Lebensqualität verbessert [24].

Wenn bei Patienten mit chronischer Anämie akute Blutverluste auftreten, ist zu beachten, dass dieselben Kompensationsmechanismen wirksam werden wie bei Patienten ohne chronische Anämie. Es gelten bei diesen Patienten daher dieselben Therapiegrundsätze und Indikationen zur Erythrozytentransfusion wie bei Patienten ohne vorbestehende chronische Anämie.

Patienten mit einer chronischen Anämie infolge primärer oder sekundärer Knochenmarkinsuffizienz sollten grundsätzlich so wenig wie möglich transfundiert werden, insbesondere, wenn eine spätere Knochenmark- bzw. Stammzelltransplantation infrage kommt. Es liegen bezüglich der Erythrozytentransfusionen bei diesen Patientengruppen keine Daten vor. Sie werden jedoch wie schwerkranke Patienten behandelt. Der Transfusionstrigger liegt bei Hämoglobinkonzentrationen $<8-7 \mathrm{~g} / \mathrm{dl}(<5,0-4,3 \mathrm{mmol} / \mathrm{l})$. Der Transfusionsbedarf kann durch Erythropoetingaben zum Teil reduziert werden. Nach derzeitigem Kenntnisstand kann Erythropoetin bei Patienten mit malignen Erkrankungen negative Wirkungen zeigen, daher sollte die Anwendung auf Patienten unter Chemotherapie beschränkt bleiben.

Für die Behandlung von Patienten mit nicht immunologisch bedingten, hämolytischen Anämien gelten dieselben Grundsätze wie bei Anämien infolge von Bildungsstörungen.

\section{Indikation zur Transfusion in der Pädiatrie und Neonatologie \\ $\nabla$}

Empfehlungen zur Transfusionsindikation und -dosierung unterschiedlicher Blutprodukte wurden erstmal in dieser Umfänglichkeit im Jahr 2002 von Roseff et al. zusammengestellt [26]. Sie gelten für Kinder nach dem 4. Lebensmonat unverändert bis heute und definieren, mit Ausnahme bei schwerer kardialer und pulmonaler Erkrankung, einen Hämatokrit von $<24 \%$ (HbWert von $8 \mathrm{~g} / \mathrm{dl}$ ) als Transfusionstrigger ( $\bullet$ Tab. 13). Jüngere Studie zeigen, analog zu Erwachsenen, dass ebenfalls bei dieser Patientengruppe aus einer restriktiven Transfusionsindikation ( $\mathrm{Hb}$ $<7 \mathrm{~g} / \mathrm{dl}$ ) verglichen mit einem liberalen Transfusionsregime $(\mathrm{Hb}<9,5 \mathrm{~g} / \mathrm{dl}$ ) keine gesundheitlichen Nachteile resultieren. Bei Kindern (Alter 3 Tage bis 14 Jahre) auf Intensivstationen zeigte sich, dass die restriktive Transfusionsindikation zu einer niedrigeren Transfusionsrate führte, ohne dass negative Einwirkungen auf den klinischen Verlauf zu verzeichnen waren [27]. Es wurden keine Unterschiede hinsichtlich der Mortalität, progressiver multipler Organdysfunktionen, nosokomialen Infekten und der Beatmungsdauer festgestellt.

Dies gilt nicht für Kinder mit Hypoxämie, instabilen Kreislaufverhältnissen, akutem Blutverlust und zyanotischen Herzvitien, auch wenn in ersten Studien an kleinen Fallzahlen $(n=125)$ bei Kindern, die sich einer Herzoperation unterziehen mussten, vergleichbare Ergebnisse erhoben werden konnten [28]. Bei diesen Patienten sollte ein Hämatokritwert von $\geq 30 \%$ angestrebt werden.

Ebenfalls hiervon ausgenommen sind Früh- oder Neugeborene bis zum 4. Lebensmonat. Die von Roseff im Jahr 2002 definierten Empfehlungen werden, abhängig vom Lebensalter, hinsichtlich der Transfusionstrigger Hämatokrit, Beatmung und Sauerstoffbedarf sowie der klinischen Symptomatik in den aktuellen Leitlinien weiter präzisiert ( $\bullet$ Tab. 14).

Vergleichsstudien im Hinblick auf restriktives vs. liberales Transfusionsschema kommen zu unterschiedlichen Ergebnissen. So zeigt sich, dass bei liberaler Erythrozytenkonzentrattransfusion chronische Lungenerkrankungen und Retinopathie häufiger auftreten. Dem gegenüber steht die Feststellung, dass eine restriktive Indikationsstellung mit häufigeren, neurologischen Komplikationen [29] und einer höheren Frequenz an Apnoe einhergeht [30].

Tab. 13 Indikation zur Transfusion bei Kindern > 4. Lebensmonat [3].

Präoperative Anämie und ein Hämatokrit < 24\%

Blutverlust $\geq 25 \%$ des Blutvolumens

Symptomatische Anämie und Hämatokrit < 24\%

Chemotherapie und/oder Radiotherapie und ein Hämatokrit < 24\%

Schwere kardiale oder pulmonale Erkrankungen und ein Hämatokrit < 40\%

Symptomatische Sichelzellanämie oder andere hereditäre Anämie 
Tab. 14 Indikation zur Transfusion bei Früh-/Neugeborenen und Säuglingen bis zum 4. Lebensmonat [3].

\begin{tabular}{|llll}
\hline Alter (Tage) & $\begin{array}{l}\text { mittlerer Hämato- } \\
\text { krit-Normwert (\%) }\end{array}$ & $\begin{array}{l}\text { Transfusionsindikation: } \\
\text { Hämatokrit-Grenze und/oder Indikationsliste }\end{array}$ \\
\hline 1 & 56 & $<40$ & - Beatmung, $\mathrm{O}_{2}$-Bedarf $\left(\mathrm{FiO}_{2}\right)>0,4$ oder \\
\hline$<15$ & 50 & $<35$ & $\begin{array}{l}\text { lebensbedrohliche Symptome durch } \\
\text { Anämie und/oder Hypovolämie }\end{array}$ \\
\hline $15-28$ & 45 & $<30$ & $\begin{array}{l}\text { - lebensbedrohliche Symptome durch } \\
\text { Anämie und/oder Hypovolämie }\end{array}$ \\
\hline$>28$ & 40 & $<25$ & - geplante Operationen \\
\hline
\end{tabular}

Die Gabe von Erythrozytenkonzentraten bei Früh- oder Neugeborenen kann vermieden werden, wenn in der 1. Woche nach der Geburt mit der Applikation von Erythropoetin in Kombination mit oraler Eisensubstitution sowie Vitamin $B_{12}$ und Folsäure begonnen wird. Die Gabe von Erythropoetin wird auch bei Kindern mit malignen Erkrankungen und Chemotherapie empfohlen. Das Ziel, die Hypoxämie bei Früh- oder Neugeborenen durch die Transfusion zu verbessern und somit die Beatmung zu reduzieren, wird oft nicht erreicht [29], so dass - analog zu Erwachsenen - ebenfalls bei dieser Patientenklientel die Forderung gestellt wird, die Transfusionstrigger durch weitere Studie kritisch zu überprüfen und den Trigger „Sauerstoffbedarf $\left(\mathrm{FiO}_{2}[\right.$ inspirierte Sauerstofffraktion] >0,4)“ zu überdenken.

Die Transfusion von länger gelagerten Erythrozytenkonzentraten zeigt ein erhöhtes Risiko für multiple Organdysfunktionen [31]. Die aktuellen Leitlinien empfehlen bei bestimmten Behandlungen, z. B. Austauschtransfusion, nur kurz gelagerte Erythrozytenkonzentrate zu verwenden [3].

\section{Durchführung und Dokumentation der Transfusion}

$\nabla$

Die Durchführung einer Transfusion erfolgt nach der Aufklärung über die Transfusion und deren Risiken durch den zuständigen Arzt sowie die Einwilligung des Patienten zu dieser medizinischen Intervention.

Ist der Patient nicht geschäftsfähig, bspw. im Fall einer bestehenden Betreuung, muss der gesetzliche Vertreter über die geplante Transfusion aufgeklärt werden. Eingeschränkt ist der Umfang dieser Aufklärungspflicht im dokumentierten Notfall.

Ist eine Aufklärung des Patienten vor der Anwendung von Blutprodukten nicht möglich, z. B. in einer Notfallsituation, dann ist der Patient nachträglich über die stattgefundene Anwendung und insbesondere die Infektions-, ggf. die Immunisierungsrisiken, aufzuklären (Sicherungsaufklärung).

Vor der Transfusion muss eine eindeutige Sicherung der Patientenidentität erfolgen, indem der Patient nach Vor- und Nachname sowie Geburtsdatum gefragt wird. Diese Angaben werden mit den Angaben des Blutpräparatebegleitscheins abgeglichen. Weitere produktbezogene Angaben auf diesem Dokument müssen mit dem Kreuzprobenetikett des Erythrozytenkonzentrats abgeglichen werden. Darüber hinaus ist die Kreuzprobenhaltbarkeit, das Ergebnis der Kreuzprobe, die Produkthaltbarkeit sowie die Unversehrtheit des Erythrozytenkonzentrats zu überprüfen. Zudem sind Angaben auf immunhämatologische Besonderheiten, z. B. das Vorliegen von Alloantikörpern und entsprechend die Bereitstellung antigennegativ getesteter Erythrozytenkonzentrate, zu prüfen.

Gleiches gilt für zusätzliche Produktbesonderheiten wie z. B. die Bestrahlung des EK.

Vor der Transfusion wird aus einer frisch entnommenen Patientenblutprobe am Bett der Bedside-Test durchgeführt, dessen Ergebnis mit der Patientenblutgruppe auf dem Begleitschein und der Präparateblutgruppe abgeglichen werden muss [1].

Das Blutgruppenergebnis des BST mit Datum und Unterschrift des Arztes muss in der Patientenakte dokumentiert werden.

Nur wenn eine Blutgruppenübereinstimmung bzw. -kompatibilität vorliegt, darf die Transfusion durchgeführt werden.

Für die Transfusion muss ein Standardtransfusionsbesteck mit Standardfilter verwendet werden. Ein Infusionsbesteck ist nicht zulässig. Die Beimischung von Medikamenten über den gleichen Zugang ist zwingend zu vermeiden.

Die Transfusionsgeschwindigkeit sollte dem klinischen Zustand des Patienten angepasst werden. Bei kreislaufstabilen, erwachsenen Patienten mit einer hochgradigen Anämie können bei Bedarf bis zu 4 Erythrozytenkonzentrate (entsprechend etwa $1000 \mathrm{ml}$ ) in 3-4 Stunden übertragen werden. Eine zu hohe Transfusionsgeschwindigkeit birgt das Risiko einer TACO.

- Sofern der transfundierende Arzt die Aufklärung nicht selber vorgenommen hat, muss er sich davon überzeugen, dass das Aufklärungsgespräch geführt wurde. Die Dokumentation hierüber, vom Arzt und Patienten unterschrieben, sowie die Einwilligung des Patienten müssen vorliegen.

- Der Bedside-Test ist der entscheidende Schritt, um eine Patientenverwechslung und somit das Risiko einer A-B-0-Verwechslung auszuschließen.

- Die Transfusionsgeschwindigkeit sollte dem klinischen Zustand des Patienten angepasst werden. 
Tab. 15 Meldungswege bei transfusionsassoziierten Nebenwirkungen [1].

\begin{tabular}{ll}
$\begin{array}{l}\text { Ereignis } \\
\begin{array}{l}\text { unerwünschte Ereignisse, alle unerwarteten } \\
\text { Komplikationen, auch Fehltransfusionen }\end{array}\end{array}$ & $\begin{array}{l}\text { Meldung an ... } \\
\text { einrichtungsintern an im Qualitätssicherungssystem fest- } \\
\text { gelegte Personen (Transfusionsverantwortlicher, Trans- } \\
\text { fusionsbeauftragter, Qualitätssicherungsbeauftragter) }\end{array}$ \\
\hline $\begin{array}{l}\text { V.a. unerwünschte Reaktionen/Nebenwirkungen } \\
\text { (UAW) beim bestimmungsgemäßen Gebrauch } \\
\text { auftretend }\end{array}$ & - pharmazeutischer Unternehmer \\
\hline $\begin{array}{l}\text { V.a. schwerwiegende unerwünschte Reaktionen/ } \\
\text { tödliche oder lebensbedrohliche Nebenwirkun- } \\
\text { gen, Behinderung, Invalidität, stationäre Behand- } \\
\text { lung (oder Verlängerung) }\end{array}$ & P pharmazeutischer Unternehmer \\
\hline
\end{tabular}

Tab. 16 Dokumentation einer Transfusion in der Krankenakte.

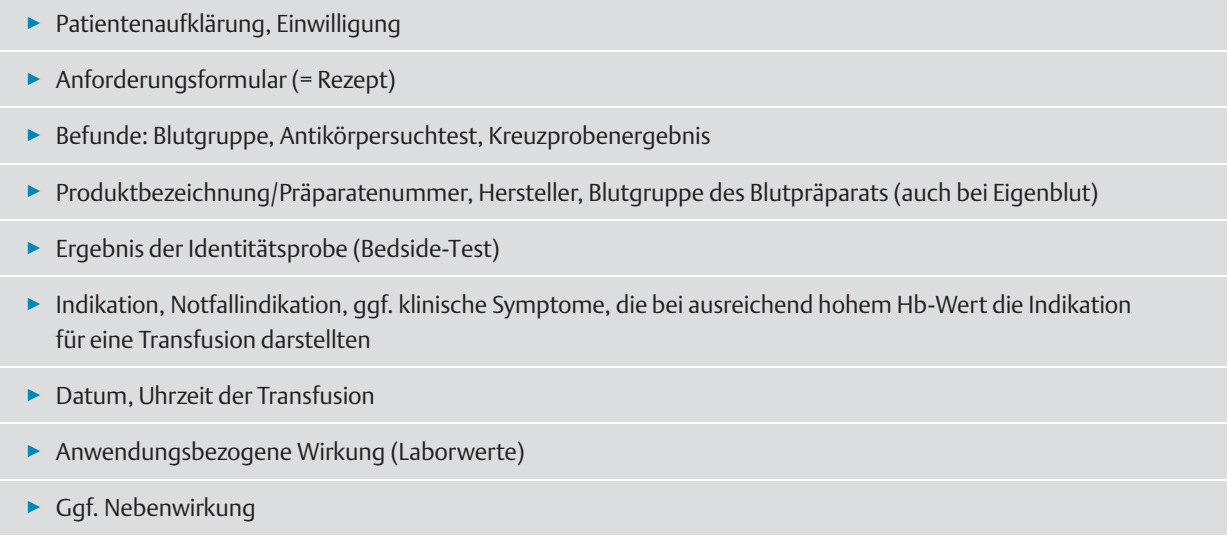

Hb: Hämoglobin

- Eine Erwärmung gekühlter Erythrozytenkonzentrate ist in der Regel nicht erforderlich.

- Die Daten der patienten- und produktbezogenen Dokumentation müssen über 30 Jahre archiviert werden.
Eine Erwärmung gekühlter Erythrozytenkonzentrate ist in der Regel nicht erforderlich. Ausnahmen sind Massivtransfusionen mit Zufuhr von mehr als $50 \mathrm{ml}$ pro Minute, bereits vor der Transfusion unterkühlte Patienten, Patienten mit einer chronischen Kälteagglutininkrankheit und hochtitrigen Kälteantikörpern oder Patienten, die auf den Kältereiz durch gekühltes Blut mit einem Vasospasmus reagieren. Zur Bluterwärmung dürfen nur für diesen Zweck zugelassene Geräte eingesetzt werden.

Eine Zwischenlagerung des Erythrozytenkonzentrats außerhalb der Blutbank oder des Blutdepots sollte weitestgehend vermieden werden. Sollte diese z. B. aufgrund einer akuten Intervention am Patienten unumgänglich sein, darf das Erythrozytenkonzentat nicht in einem allgemeinen Stationskühlschrank zwischengelagert werden.

Eröffnete („angestochene“) Erythrozytenkonzentrate sind innerhalb von 6 Stunden zu transfundieren.

Die Blutbeutel von transfundierten Blutprodukten müssen für 24 Stunden bei $4 \pm 2{ }^{\circ} \mathrm{C}$ asserviert werden, da im Fall einer Transfusionsnebenwirkung weitere Untersuchungen aus dem Restblut im Beutel durchgeführt werden.

Im $\S 16$ TFG (Transfusionsgesetz) ist festgehalten, dass im Zusammenhang mit der Anwendung von Blutprodukten aufgetretene, unerwünschte Ereignisse oder Nebenwirkungen meldepflichtig sind. Eine Nebenwirkung setzt einen bestimmungsgemäßen Gebrauch voraus. Jede transfundierende Einrichtung muss deshalb hausintern Meldewege unter Einbeziehung des Transfusionsbeauftragten und -verantwortlichen etabliert haben ( Tab. 15).

Die Einrichtung der Krankenversorgung hat sicherzustellen, dass die Daten der patienten- und produktbezogenen Dokumentation über 30 Jahre archiviert und im Fall einer Rückverfolgung unverzüglich zur Verfügung gestellt werden können (§14 Abs. 2 und 3 TFG).

Patientenbezogene Daten müssen in der Krankenakte abgelegt werden ( Tab. 16). 


\section{Fazit}

$\nabla$

Die Indikation zur Transfusion von Erythrozytenkonzentraten bzw. allgemein Blutprodukten sollte auf Basis der jeweiligen Richtlinien und unter Berücksichtigung des individuellen Gesundheits- und Allgemeinzustands des Patienten getroffen werden. Denn auch wenn Blutprodukte so sicher sind wie nie, bleiben mögliche transfusionsassoziierte Risiken durch die Gabe von allogenem Blut. Ein leitlinienkonformes Vorgehen sichert die Blutversorgung als gemeinsame Aufgabe von Transfusionsmedizinern und klinischen Fachvertretern. Kliniken, die nicht direkt an eine Blutbank angeschlossen sind, haben entsprechende Maßnahmen zu ergreifen, um eine zeitgerechte Versorgung mit Blutsprodukten sicherstellen zu können.

\section{Interessenkonflikt}

$\nabla$

Die Autoren erklären, dass kein Interessenkonflikt besteht.

\section{Literatur}

$\nabla$

1 Bundesärztekammer. Bundesärztekammer im Einvernehmen mit dem PEI: Richtlinien zur Gewinnung von Blut und Blutbestandteilen und zur Anwendung von Blutprodukten (Hämotherapie), Gesamtnovelle 2005 mit Richtlinienanpassung 2010. Köln: Deutscher Ärzteverlag; 2010

2 Funk M, Günay S. Hämovigilanzbericht des Paul-Ehrlich-Institutes 2010. Im Internet: pharmakovigilanz2@pei.de

3 Bundesärztekammer. Querschnitts-Leitlinien zur Therapie mit Blutkomponenten und Plasmderivaten. 4. Auf. Köln: Deutscher Ärzteverlag; 2009

4 Bennett-Guerrero E, Veldman TH, Doctor A et al. Evolution of adverse changes in stored RBCs. PNAS 2007; 104: $17063-17068$

5 Koch CG, Li L, Sessler DI et al. Duration of red-cell storage and complications after cardiac surgery. N Engl J Med 2008; 358: 1229-1239

6 Edgren G, Kamper-Jørgensen M, Eloranta $S$ et al. Duration of red blood cell storage and survival of transfused patients (CME). Transfusion 2010; 50: 1185 - 1195; Erratum in: Transfusion 2010; 50: 1857

7 Wang D, Sun J, Solomon SB et al. Transfusion of older stored blood and risk of death: a meta-analysis. Transfusion 2012; 52: $1184-1195$

8 Karkouti K, Stukel TA, Beattle WS et al. Relationship of erythrocyte transfusion with short- and long-term mortality in a population-based surgical cohort. Anesthesiology 2012; 117: 1175-1183

9 Saager L, Turan A, Dalton JE et al. Erythrocyte storage duration is not associated with increased mortality in noncardiac surgical patients. Anesthesiology [im Druck]

10 Atzil S, Arad M, Glasner A et al. Blood transfusion promotes cancer progression: a critical role for aged erythrocytes. Anesthesiology 2008; 108: 989-997

11 Gombotz H, Rehak PH, Shander A et al. Blood use in elective surgery: the austrian benchmark study. Transfusion 2007; 47: $1468-1480$

12 Hébert PC, Van der Linden P, Biro G et al. Physiologic aspects of anemia. Crit Care Clin 2004; 20: 187-212

13 Carson JL, Grossman BJ, Kleinman $S$ et al. Red blood cell transfusion: a clinical practice guideline from the AABB. Ann Intern Med 2012; 157: 49-58

14 Carson JL, Terrin ML, Noveck $H$ et al. Liberal or restrictive transfusion in high-risk patients after hip surgery. N Engl J Med 2011; 365: 2453-2462

15 Walsh TS, McClelland DB, Lee RJ et al. Prevalence of ischaemic heart disease at admission to intensive care and its influence on red cell transfusion thresholds: multicentre Scottish Study. Br J Anaesth 2005; 94: 445 - 452

16 Deutsche Gesellschaft für Unfallchirurgie. S3-Leitlinie Polytrauma/Schwerverletzten-Behandlung, AWMF Register-Nr.012/019. Im Internet: http://www.awmf.org/uploads/tx_szleitlinien/012-019m_S3_Polytrauma_Schwerverletzten-

Behandlung_2011-07.pdf; Stand: 15.12.2012

17 Shander A, Javidroozi M, Ozawa S et al. What is really dangerous: anaemia or transfusion? Br J Anaesth 2011; 107 (Suppl. 1): i41-i59

18 Marik PE, Corwin HL. Efficacy of red blood cell transfusion in the critically ill: a systematic review of the literature. Crit Care Med 2008, 36: 2667-2674

19 Glance LG, Dick AW, Mukamel DB et al. Association between intraoperative blood transfusion and mortality and morbidity in patients undergoing noncardiac surgery. Anesthesiology 2011; 114: 283-292

20 Karkoutin K, Wijeysundera DN, Yau TM et al. Advance targeted transfusion in anemic cardiac surgical patients for kidney protection. Anesthesiology 2012; 116: 13-21

21 Hébert PC, Wells G, Martin C et al. Variation in red cell transfusion practice in the intensive care unit: a multicentre cohort study. Crit Care 1999; 3: 57-63

22 Hajjar LA, Vincent JL, Galas FR et al. Transfusion requirements after cardiac surgery: the TRACS randomized controlled trial. JAMA 2010; 13: 1559-1567

23 Carson JL, Carless PA, Hebert PC. Transfusion thresholds and other strategies for guiding allogeneic red blood cell transfusion. Cochrane Database Syst Rev 2012; 4: CD002042

24 Horwich TB, Fonarow GC, Hamilton MA et al. Anemia is associated with worse symptoms, greater impairment in functional capacity and a significant increase in mortality in patients with advanced heart failure. J Am Coll Cardiol 2002; 39: 1780-1786 
25 Drüeke TB, Locatelli F, Clyne N et al. Normalization of hemoglobin level in patients with chronic kidney disease and anemia. N Engl J Med 2006; 16: 2071 - 2084

26 Roseff SD, Luban NLC, Manno CS. Guidelines for assessing appropriateness of pediatric transfusion. Transfusion 2002; 42: 1398-1413

27 Lacroix J, Hébert PC, Hutchison JS et al. Transfusion strategies for patients in pediatric intensive care units. N Eng J Med 2007; 356: 1609-1619

28 Willems A, Harrington K, Lacroix J et al. Comparison of two red-cell transfusion strategies after pediatric cardiac surgery: a subgroup analysis. Crit Care Med 2010; 38: 649-656

29 Kasat K, Hendricks-Munoz KD, Mally PV. Neonatal red blood cell transfusions: searching for better guidelines. Blood Transfus 2011; 9: 86-94

30 Bell EF, Strauss RG, Widness JA et al. Randomized trial of liberal versus restrictive guidelines for red blood cell transfusion in preterm infants. Pediatrics 2005; 115: 1685-1691

31 Gauvin F, Spinella PC, Lacroix J et al. Association between length of storage of transfused red blood cells and multiple organ dysfunction syndrom in pediatric intensive care patients. Transfusion 2010; 50: 1902 - 1913

32 Schmidt M, Sireis W, Seifried E et al. Sicherheit der Blutprodukte - Update 2011. Transfusionsmedizin 2011; $1: 28-50$

\section{Impressum CME - Transfusionsmedizin}

$\nabla$

(c) 2013 Georg Thieme Verlag KG, Rüdigerstraße 14, 70469 Stuttgart

Unsere Homepage:

http://www.thieme.de

Printed in Germany

Satz: Ziegler + Müller, text form files, Kirchentellinsfurt

Druck: F\&W Mediencenter GmbH, Kienberg

Redaktionelle Mitarbeit: Sabine M. Rüdesheim, Frechen-Königsdorf

Ansprechpartner: Dr. Silvia Jung

Redaktion

Georg Thieme Verlag KG

Klinik und Praxis

E-Mail: Silvia.Jung@thieme.de
Die CME-Beiträge der CME-Fortbildung in Transfusionsmedizin wurden durch die Nordrheinische Akademie für ärztliche Fort- und Weiterbildung anerkannt. Die Transfusionsmedizin ist zur Vergabe der Fortbildungspunkte für diese Fortbildungseinheit berechtigt. Diese Fortbildungspunkte der Nordrheinischen Akademie für ärztliche Fort- und Weiterbildung werden von den anderen zertifizierenden Ärztekammern sowie, gemäß der Novellierung der DFPRichtlinien vom 30.06.2010 (§14 Ziff. 3), auch von den österreichischen Ärztekammern anerkannt. Die Vergabe der Fortbildungspunkte ist nicht an ein Abonnement gekoppelt!

Für Angaben über Dosierungsanweisungen und Applikationsformen kann vom Verlag keine Gewähr übernommen werden. Jeder Benutzer ist angehalten, durch sorgfältige Prüfung der Beipackzettel der verwendeten Präparate und ggf. nach Konsultation eines Spezialisten festzustellen, ob die dort gegebene Empfehlung für Dosierungen oder die Beachtung von Kontraindikationen gegenüber der Angabe in dieser Broschüre abweicht. Eine solche Prüfung ist besonders wichtig bei selten verwendeten Präparaten oder solchen, die neu auf dem Markt gebracht worden sind. Jede Dosierung oder Applikation erfolgt auf eigene Gefahr des Benutzers. 


\section{CME-Fragen Rationale Indikation \\ zur Transfusion von \\ Erythrozytenkonzentraten}

1 Welche Aussage zu restriktiven Transfusionsindikationen trifft zu?

A Sie sehen eine Erythrozytentransfusion erst bei Unterschreiten des kritischen Hämatokrits vor.

B Sie sind altersabhängig nur bei jüngeren Patienten anzuwenden.

C Sie gehen bei den meisten Patienten nicht mit erhöhten Komplikationsraten einher.

D Sie basieren auf Erkenntnissen aus retrospektiven Analysen und können daher nur mit niedrigem Evidenzgrad empfohlen werden.

E Restriktive Transfusionsregime sind bei Patienten mit Risikofaktoren kontraindiziert.

2 Welche Aussage trifft nicht zu? Zu klinischen Symptomen, die auf eine anämische Hypoxie hinweisen können, gehören:

A Tachykardie

B Tachypnoe

C Bewusstseinsstörungen

D Laktatazidose

E Anstieg der zentralvenösen Sauerstoffsättigung > 70\%

3 Welche Aussage trifft nicht zu? Welche Faktoren müssen neben der $\mathrm{Hb}$-Konzentration bei der Indikation zur Transfusion berücksichtigt werden?

A Ursache, Dauer, und Schweregrad der Anämie

B Ausmaß und Geschwindigkeit des Blutverlusts

C Geschlecht des Patienten

D Symptome einer anämischen Hypoxie (physiologische Transfusionstrigger)

E Zeichen einer Hypovolämie

4 Welche Aussage trifft nicht zu? Welche physiologischen Kompensationsmechanismen sind bei akuter normovolämischer Anämie wirksam?

A Steigerung des Herzschlagvolumens

B Steigerung der Herzfrequenz

C Verstärkte Sauerstoffausschöpfung aus dem venösen Blut

D Umverteilung der Durchblutung im Organismus

E Zunahme des Herzzeitvolumens

5 Setzen Sie die geschätzten Häufigkeiten für folgende Transfusionsrisiken nach Erythrozytenkonzentratgabe in Deutschland in eine logische Reihenfolge von häufig bis selten:

1. humanes Immundefizienzvirus

2. Hepatitis-B-Virus

3. ABO-Verwechslung

4. bakterielle Sepsis

A $1,2,4,3$

B $3,1,2,4$

C $4,3,1,2$

D $3,4,2,1$

E $3,2,1,4$
6 Welche Aussage zur Lagerung von Erythrozytenkonzentraten trifft nicht zu?

A Erythrozytenkonzentrate müssen bei $4^{\circ} \mathrm{C}$ gelagert werden.

B Erythrozytenkonzentrate müssen nach Unterbrechung der Kühlkette innerhalb von 6 Stunden transfundiert werden.

C Falls sich die Transfusion auf Station verzögert, sollte das Erythrozytenkonzentrat bis zur Transfusion im Stationskühlschrank aufbewahrt werden.

D Bei einer allogenen Erythrozytentransfusion muss kein Bedside-Test aus dem Präparat vor der Transfusion durchgeführt werden.

E Die Transfusion eines Erythrozytenkonzentrats führt zu einem $\mathrm{Hb}$-Anstieg von ca. 1 -1,5 g/dl.

7 Welche Aussage trifft für die Transfusion von Erythrozytenkonzentraten zu?

A Bei einem $\mathrm{Hb}$-Wert von $100 \mathrm{~g} / \mathrm{l}$ sollten Patienten mit einer KHK vor Operationen Erythrozytenkonzentrate erhalten.

B Da die Anämietoleranz im Alter abnimmt, sollten ältere Patienten auch bei adäquaten Kompensationsmechanismen ab Hb-Werten von ca. $80 \mathrm{~g} / 1$ immer Erythrozytenkonzentrate erhalten.

C Unter einem $\mathrm{Hb}$-Wert $<60 \mathrm{~g} / \mathrm{l}$ sollte eine Transfusion erfolgen.

D Es gibt für Patienten ohne Risikofaktoren keinen kritischen Hb-Wert, ab dem eine absolute Transfusionsindikation besteht.

E Um das Morbiditäts- und Mortalitätsrisiko bei intensivpflichtigen Patienten zu reduzieren, sollten bei diesen ein Ziel-Hb-Wert von ca. $120 \mathrm{~g} / \mathrm{l}$ angestrebt werden.

8 Folgende Erkrankung bzw. Situation stellt keine Indikation zur Bestrahlung transfundierter Erythrozytenkonzentrate dar:

A Morbus Hodgkin

B Z.n. allogener Stammzelltransplantation

C intrauterine Transfusion

D Non-Hodgkin-Lymphom

E Morbus Werlhof 
Welche Aussage trifft zu? Treten im Zusammenhang mit der Anwendung von Blutprodukten unerwünschte Ereignisse auf, so hat der behandelnde Arzt ...

1. festzulegen, ob es sich um eine schwerwiegende Reaktion handelt.

2. immer den Hersteller des Blutprodukts

(z. B. Blutspendedienst) zu informieren.

3. immer das Paul-Ehrlich-Institut (Langen) zu informieren.

4. seinen Transfusionsbeauftragten zu informieren.

5. immer die Arzneimittelkommission der deutschen Ärzteschaft zu informieren.

A Alle Antworten sind richtig.

B $1,2,3$ und 4 sind richtig.

C 1,2 und 4 sind richtig.

D $1,2,4$ und 5 sind richtig.

E 2, 4 und 5 sind richtig.
Welche Aussage trifft zu? Welche Angaben müssen im Zusammenhang mit der Transfusion in der Patientenakte dokumentiert werden?

1. die Risikoaufklärung und Einwilligung des Patienten 2. die Indikation zur Transfusion

3. das Ergebnis des AB0-Identitätstests (Bedside-Test)

4. Datum und Uhrzeit der Verabreichung von Blutprodukten

5. Wirkung bzw. Nebenwirkungen

A Alle Aussagen sind richtig.

B Die Aussagen 1, 3 und 5 sind richtig.

C Die Aussagen 1, 2, 3 und 5 sind richtig.

D Die Aussagen 1, 3 und 4 sind richtig.

E Die Aussagen 1 und 3 sind richtig.

- Viel Erfolg bei lhrer CME-Teilnahme unter http://cme.thieme.de

- Diese Fortbildungseinheit ist in der Regel 12 Monate online für eine CME-Teilnahme verfügbar. Bitte informieren Sie sich über die genaue Gültigkeit unter http://cme.thieme.de

- Sollten Sie Fragen zur Online-Teilnahme haben, unter http://cme.thieme.de/hilfe finden Sie eine ausführliche Anleitung. 